\title{
Research and Development of Butterfly Microfluidic Gene Chip for 19 Common Pathogenic Microorganisms of Nosocomial Infection
}

\author{
Jingjing Yu, Xu Zhang, Jinhai Tian, Rong Ma, Qi Huang, Jia Cao, Jia Wang, Libin Wang* \\ The General Hospital of Ningxia Medical University, Department of Beijing National Biochip Research Center sub- \\ center in Ningxia, Yinchuan 750004, Ningxia Hui Autonomous Region, China
}

*Corresponding author: Libin Wang, wanglibin007@126.com

Copyright: (C) 2022 Author(s). This is an open-access article distributed under the terms of the Creative Commons Attribution License (CC BY 4.0), permitting distribution and reproduction in any medium, provided the original work is cited.

\begin{abstract}
Based on butterfly microfluidic gene chip technology, a method for rapid, accurate and efficient detection of 19 common pathogenic microorganisms of nosocomial infection was established, and a butterfly microfluidic gene chip with high-throughput detection was designed and fabricated. Using constant temperature amplification technology, using the polymerase with chain replacement function to react at constant temperature $\left(65^{\circ} \mathrm{C}\right)$ and combined with microfluidic chip technology, primers were designed according to the target genes of 19 pathogenic microorganisms, and a butterfly microfluidic gene chip which can detect 19 common pathogenic microorganisms of nosocomial infection was made to simplify the inspection operation process and verify the sensitivity of the chip. The butterfly microfluidic gene chip can be used for the rapid and efficient detection of 19 common pathogenic microorganisms of nosocomial infection, and provides a new idea for the detection and auxiliary diagnosis of pathogenic microorganisms of nosocomial infection.
\end{abstract}

Keywords: Nosocomial infection; Butterfly microfluidic gene chip; High throughput

Online publication: January 19, 2022

\section{Introduction}

Nosocomial infection, also known as hospital acquired infection, in a narrow sense, refers to any clearly diagnosed infection or disease caused by an inpatient who does not exist at the time of admission and is in the incubation period but suffers from pathogen invasion during hospitalization, regardless of whether the infected person develops symptoms after hospitalization or after discharge. Nosocomial infection increases the social and economic burden, causing physical and mental pain and economic losses to patients. Literature data shows that there are about 18 million cases of blood flow infection in the world every year, with a mortality of 20-50\%. About 20000 people die of blood flow infection every day ${ }^{[1]}$. Nosocomial infection has become a major clinical problem in the medical field, and its situation is grim and not optimistic.

In this study, the mature butterfly microfluidic gene chip technology was used to produce a butterfly microfluidic gene chip that can detect 19 common pathogenic microorganisms of nosocomial infection, which simplified the operation process of clinical microbiology test, and laid a certain foundation for its preliminary application in clinical microbiology test. The feasibility of high-throughput screening of infectious pathogenic microorganisms based on microfluidic technology was discussed ${ }^{[2]}$. 


\section{Chip design and chip detection}

\subsection{Primer design}

According to the Latin names of 19 biological microorganisms, the standard naming, species information and Taxonomy ID information of each microorganism in the database are searched from the Taxonomy database of NCBI to collect microbial sequences ${ }^{[3]}$. Among the 19 microorganisms, Acinetobacter baumanni selected gyrB gene, a highly conserved B subunit gene encoding gyrase, as the amplification target. This gene is a specific gene of Acinetobacter baumanni. Many literatures use PCR or LAMP technology to involve gene fragments. After the functional gene target sequences of other 18 microorganisms were searched, Muscle was used for sequence comparison and other database tools were used to obtain the conservative gene sequences. With the conservative gene sequences as input, primer sequences were designed by combining the ring amplified primer database and NCBI-blast. The detailed gene sequences collected are shown in Table 1. After several chip verification, some primers with nonspecific signals were screened out, and the specific signals were retained as the final primer set. All primers were synthesized by Beijing Boao Jingdian Company.

Table 1. Detailed genetic information of the microbial sequence involved

\begin{tabular}{cccc}
\hline No. & Microorganism & English name & Functional gene \\
\hline$*-69$ & Escherichia coli & Escherichia coli & uidA \\
2 & Staphylococcus aureus & Staphylococcus aureus & coa \\
3 & Klebsiella pneumoniae & Klebsiella pneumoniae & rcsA \\
4 & Acinetobacter baumannii & Acinetobacter baumannii & gyrB \\
5 & Pseudomonas aeruginosa & Pseudomonas aeruginosa & ETA \\
6 & Enterococcus faecalis & Enterococcus faecalis & efoo27 \\
7 & Enterobacter cloacae & Enterobacter cloacae & - \\
8 & Stenotrophomonas maltophilia & Stenotrophomonas maltophilia & TonB \\
9 & Streptococcus pneumoniae & Streptococcus pneumoniae & lytA \\
10 & Haemophilus influenzae & Haemophilus influenzae & pal \\
11 & Klebsiella oxytoca & Klebsiella oxytoca & rpoB \\
12 & Enterococcus faecaium & Enterococcus faecaium & mddl \\
13 & Aspergillus fumigatus & Aspergillus fumigatus & - \\
14 & Candida albicans & Candida albicans & - \\
15 & Proteus mirabilis & Proteus mirabilis & ureR \\
16 & Morganella morganii & Morganella morganii & - \\
17 & Serratia marcescens & Serratia marcescens & Luxs \\
18 & Enterobacter aerogenes & Enterobacter aerogenes & HDC \\
19 & Streptococcus pyogenes & Streptococcus pyogenes & gyrB \\
\hline
\end{tabular}

\subsection{Chip design}

Thermostatic disk microfluidic gene chip adopts thermostatic amplification technology, namely Loopmediated isothermal amplification LAMP. It uses polymerase with chain replacement function and fluorescence dye incorporation method to detect real-time fluorescence in constant temperature reaction at $65^{\circ} \mathrm{C}$. The positive sample will produce an "S" shaped amplification curve to amplify and detect the target gene in one step. The method is combined with microfluidic chip technology to complete the highthroughput detection of a variety of nucleic acid target genes. See Figure 1 for the structure of disk chip: Each chip is equipped with 24 reaction tanks, which are numbered counterclockwise, of which the inlet and outlet 1 corresponds to the No. 1 reaction tank. Each chip is embedded and fixed with a set of LAMP 
primers in a specific reaction pool for the amplification of a nucleic acid target sequence ${ }^{[4]}$. Beijing Boao Jingdian Co., Ltd. was entrusted to produce butterfly microfluidic gene detection chips for 19 common pathogenic microorganisms of hospital infection.

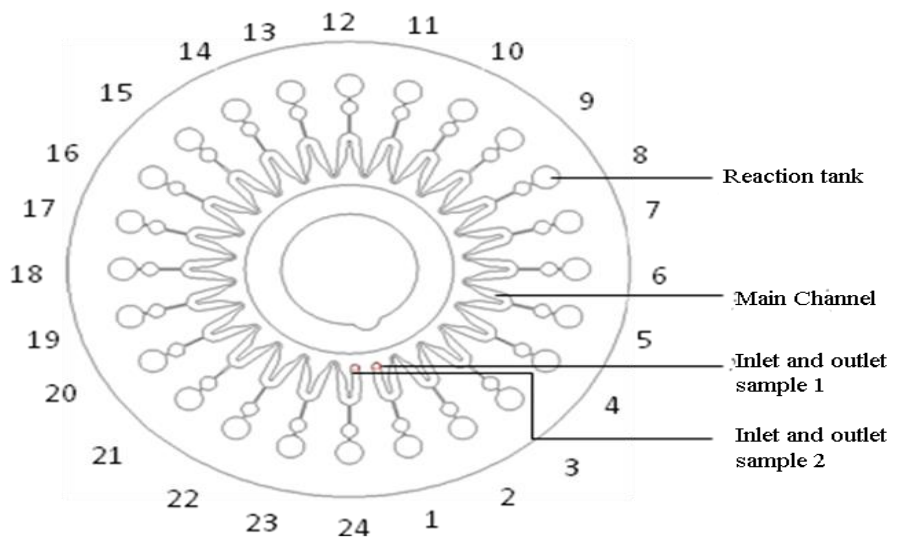

Figure 1. Structure diagram of disk microfluidic gene detection chip

\section{Experiment}

\subsection{Fabrication of microfluidic chip}

Firstly, nucleic acid was extracted from sputum, and DNA in lavage fluid was extracted using the crystal core sputum microbial DNA extraction kit - zirconium bead magnetic bead method of Boao Biological Group Co., LTD., and the nucleic acid products prepared were stored at $-80^{\circ} \mathrm{C}$.

The constant temperature amplification reagent was taken out from the kit, thawed at room temperature, shaken gently, mixed well, and centrifuged. $26.0 \mu \mathrm{L}$ thermostatic amplification reagent was added to 200 $\mu \mathrm{L}$ centrifuge tube in the system area and moved to the PCR template area. Each sample was limited to one centrifuge tube.

In the template loading area, $26.0 \mu \mathrm{L}$ of tested nucleic acid sample (or reference substance) was added to the sample area of template, mixed well, centrifuged. The total volume of each nucleic acid amplification reaction system was $52 \mu \mathrm{L}$. In the template area, the chip was taken out to restore it to room temperature, the inlet and outlet of the chip were put upward in the clean workbench, and $52 \mu \mathrm{L}$ the prepared nucleic acid amplification reaction system prepared by absorbing was added into the main channel of the chip from the sample inlet and outlet 1 . When the main channel of the chip is filled (at least 2-23 holes), stop adding samples. The sealing film was covered on the inlet and outlet to fit tightly ${ }^{[5]}$.

The chip was fixed on the low-speed centrifuge and centrifuged at 6000 RPM for 30s. RTisochip-A thermostatic amplification microfluidic chip nucleic acid analyzer was turned on and the light source was preheated for 10min. The chip was put into the detection bin, the sample information was input and run at $65^{\circ} \mathrm{C}$ for 50 minutes, and the results were interpreted through the positive reading value (Figure 2).

\subsection{Results interpretation of microfluidic chip}

After the detection, the software will automatically process and analyze the fluorescent data, "fluorescence curve area" displays the normalization curve, and the "detection result" area displays the quality control results and the detection results of each detection index. The reference value of the index to be inspected takes 40min as the limit. When the amplification time value of the detection index is less than or equal to the reference value of the index, it is judged as positive. When the amplification time value of the detection index is more than the reference value of the index, it is judged as negative. When the amplification time value of the detection index is less than or equal to the reference value of the index, it is judged as positive and the corresponding Tp value is displayed. When the amplification time value of the detection index is 
less than or equal to the reference value of the index, it is judged as positive and "-" is displayed.

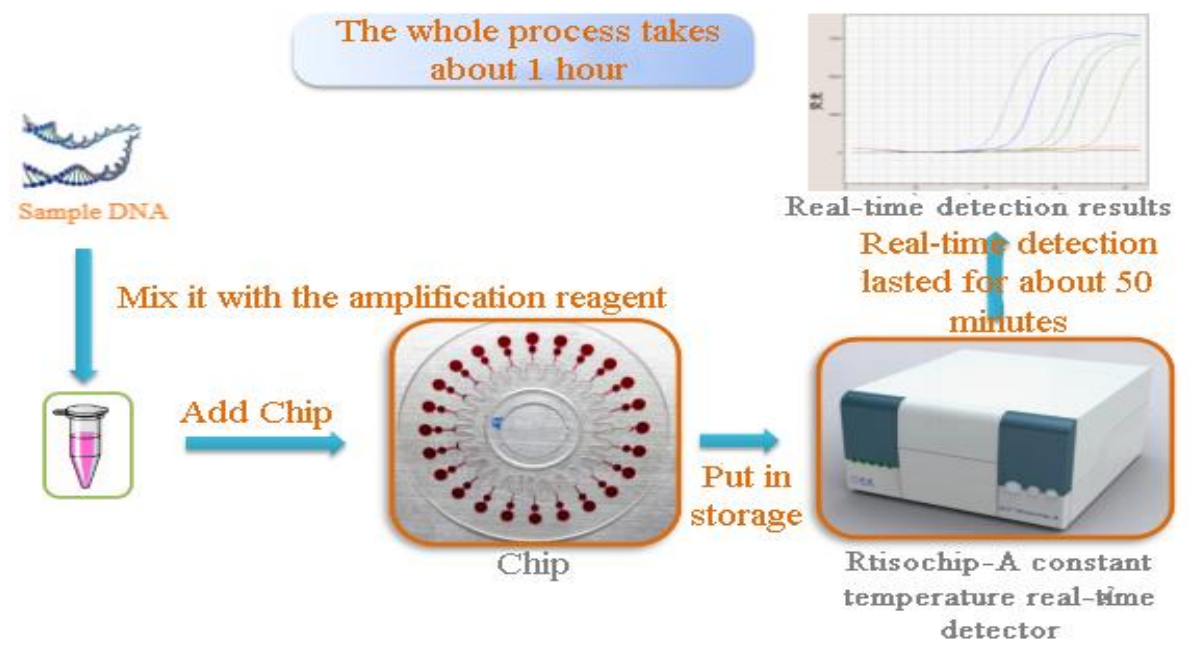

Figure 2. Detection process of disk microfluidic gene chip

\subsection{Results of microfluidic chip}

This chip (Figure 1) is the structure diagram of disk microfluidic gene detection chip. Each chip is equipped with 24 reaction tanks, which are numbered counterclockwise, of which the inlet and outlet 1 corresponds to the No. 1 reaction tank, which can detect 19 kinds of pathogenic microorganisms that have been designed. Table 3 shows the microorganisms detected by the pathogen microfluidic chip project and their abbreviations. Table 4 shows the arrangement law of pathogens in the chip reaction pool, including three groups of quality control primers (positive control PC, IC and negative control NC) and blank control BC.

Table 3. Microorganisms detected by microfluidic chip

\begin{tabular}{|c|c|c|c|}
\hline No. & Chinese name & English name & Abbreviation \\
\hline 1 & Escherichia coli & Escherichia coli & Eco \\
\hline 2 & Staphylococcus aureus & Staphylococcus aureus & Sau \\
\hline 3 & Klebsiella pneumoniae & Klebsiella pneumoniae & Kpn \\
\hline 4 & Acinetobacter baumannii & Acinetobacter baumannii & Aba \\
\hline 5 & Pseudomonas aeruginosa & Pseudomonas aeruginosa & $\mathrm{Pae}$ \\
\hline 6 & Enterococcus faecalis & Enterococcus faecalis & Efa \\
\hline 7 & Enterobacter cloacae & Enterobacter cloacae & Ecl \\
\hline 8 & Stenotrophomonas maltophilia & Stenotrophomonas maltophilia & Sma \\
\hline 9 & Streptococcus pneumoniae & Streptococcus pneumoniae & Spn \\
\hline 10 & Haemophilus influenzae & Haemophilus influenzae & Hin \\
\hline 11 & Klebsiella oxytoca & Klebsiella oxytoca & Kox \\
\hline 12 & Enterococcus faecaium & Enterococcus faecaium & Efa \\
\hline 13 & Aspergillus fumigatus & Aspergillus fumigatus & Afu \\
\hline 14 & Candida albicans & Candida albicans & $\mathrm{Cal}$ \\
\hline 15 & Proteus mirabilis & Proteus mirabilis & Pmi \\
\hline 16 & Morganella morganii & Morganella morganii & Mmo \\
\hline 17 & Serratia marcescens & Serratia marcescens & Sma \\
\hline 18 & Enterobacter aerogenes & Enterobacter aerogenes & $\mathrm{Eae}$ \\
\hline 19 & Streptococcus pyogenes & Streptococcus pyogenes & Spy \\
\hline
\end{tabular}


Table 4. LAMP primer layout of microfluidic chip

\begin{tabular}{lllllllllllll}
\hline Reaction tank & $\mathbf{1}$ & $\mathbf{2}$ & $\mathbf{3}$ & $\mathbf{4}$ & $\mathbf{5}$ & $\mathbf{6}$ & $\mathbf{7}$ & $\mathbf{8}$ & $\mathbf{9}$ & $\mathbf{1 0}$ & $\mathbf{1 1}$ & $\mathbf{1 2}$ \\
\hline Primer & PC & IC & Eco & Sau & Kpn & Aba & Pae & Efa & Ecl & Sma & Spn & Hin \\
Reaction tank & 13 & 14 & 15 & 16 & 17 & 18 & 19 & 20 & 21 & 22 & 23 & 24 \\
Primer & Kox & Efa & Afu & Cal & Pmi & Mmo & Sma & Ea & Spy & NC & BC & BC \\
\hline
\end{tabular}

\subsection{Sensitivity test results of gene chip}

The chip is set with positive quality control (PC). Figure 3 shows all 19 microorganisms. The detection amplification curve of infection samples and the detection curve of positive quality control are "S" shaped amplification curve of real-time fluorescence, that is, they are judged to be positive (each microorganism has three repetitions, and only one result is shown in the figure).

If according to the result interpretation criteria, the positive quality control test results should be positive, while the negative quality control results are negative. If any result is wrong, it is determined that the sample inspection result is invalid and needs to be rechecked. The positive internal control is a human specific primer. When clinical samples are tested, the result should be positive; If the content of human genomic DNA in the sample is small, the result may be negative, indicating that the number of human cells in the sample is small. It is recommended to re-collect the sample for detection.

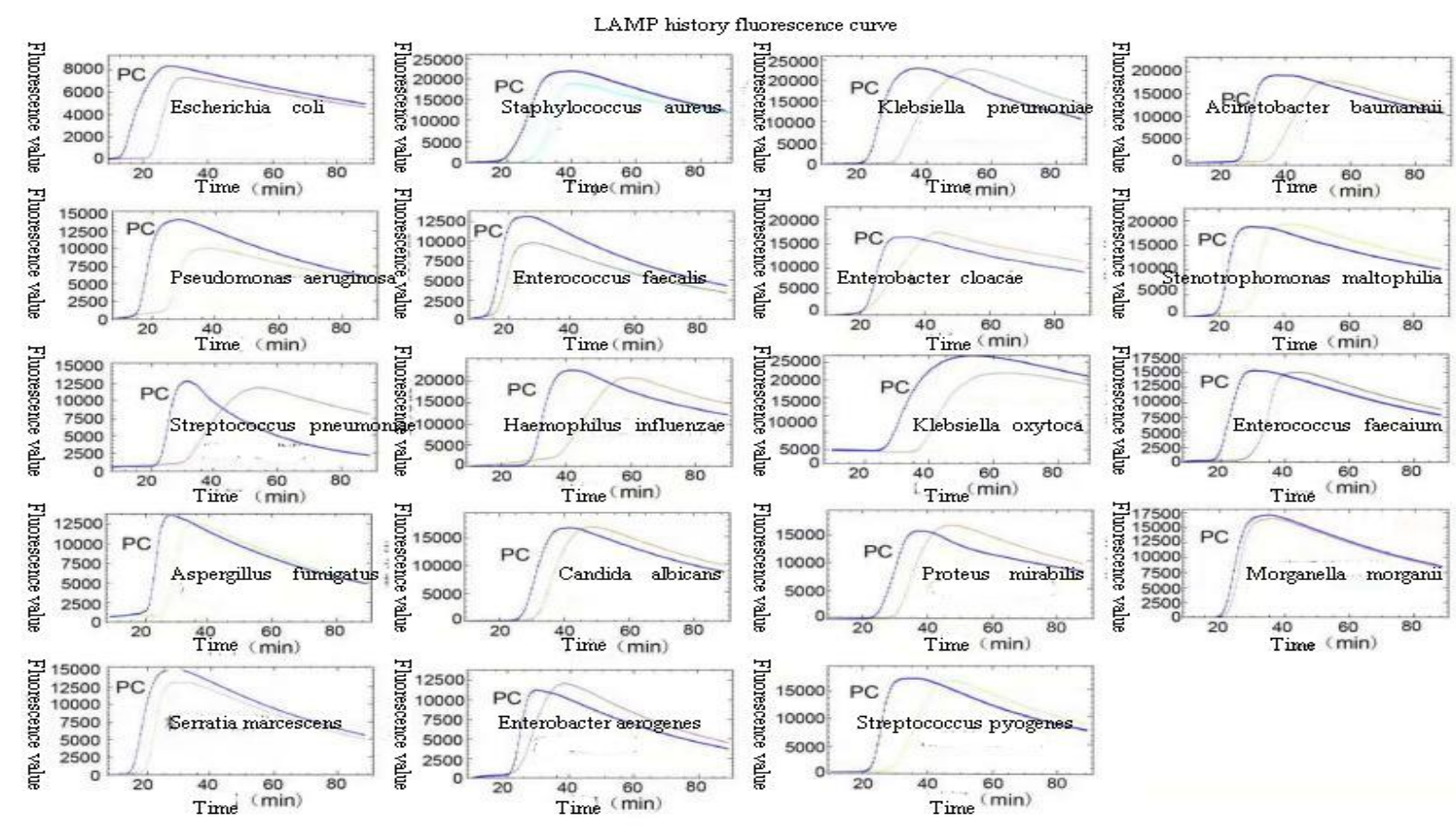

Figure 3. Experimental results of sensitivity of microfluidic chip

\section{Discussion}

In recent years, great progress has been made in the field of microfluidic chip systems. Compared with the traditional macro large volume system, it has the advantages of high efficiency, high safety factor, low loss and high sensitivity ${ }^{[6-7]}$. As a high-throughput micro scale analysis equipment, the microfluidic chip system shows great potential value in high-sensitivity detection of various biomolecules and chemistry. Compared with other detection technologies, microfluidic chip detection technology has the following advantages:

First, it can detect very small amounts of samples. Second, the reagent cost is reduced. Third, the detection flux is improved. Fourth, it has achieved a high degree of automation. Fifthly, the microfluidic 
reaction rate is improved, and the detection process is time-consuming, which ensures the accuracy, stability and repeatability of the detection results. Therefore, microfluidic gene chip technology has a good application prospect and value in the detection of pathogenic microorganisms.

In conclusion, the butterfly microfluidic gene chip which can detect 19 kinds of common pathogenic microorganisms of nosocomial infection was produced in this study, which simplifies the test operation process, has strong specificity and high sensitivity, and provides an efficient and fast method for the detection of common pathogens of nosocomial infection. The research and development of the chip can provide technical support for the prevention and control of nosocomial infection and the screening of transmission routes, provide strong support for the accurate anti infection treatment of patients with nosocomial infection, and effectively control the spread of nosocomial infection. Next, a large number of clinical tests will be carried out to further verify the chip method and strive to achieve transformation.

\section{Funding}

Key Research and Development Plan Project of Ningxia Hui Autonomous Region (Project No.: 2019BEG03026); Ningxia Overseas Returnees Innovation and Entrepreneurship Project (Project No.: 2017-659); Ningxia Key Research and Development Plan Project (Project No. 2018BFG02017); Ningxia Medical University Young Backbone Talents Training Program School-level Academic Technology Leader Reserve Cultivation Object; First-Class Discipline Construction Founded Project of Ningxia Medical University and the School of Clinical Medicine (No.NXYLXK2017A05).

\section{Disclosure statement}

The author declares no conflict of interest.

\section{References}

[1] Liu J, 2019, Thoughts on Some Problems of Bloodstream Infection. National Medical Journal of China, (31): 2471-2474.

[2] Chen Y, Ji J, Ying C, et al., 2019, Drug Resistance Monitoring Report of Bloodstream Infection Bacteria in China from 2014 to 2015. Chinese Journal of Clinical Infectious Diseases, 12(1): 24-37.

[3] Leibovici-Weissman Y, Tau N, Yahav D, 2019, Bloodstream Infections in the Elderly: What is the Real Goal?, Aging Clin, DOI: 10.1007/s40520-019-01337-w.

[4] Zhan G, Lu J, Sun J, et al., 2019, Analysis of Clinical Characteristics and Drug Resistance of Bloodstream Infection Caused by Escherichia Coli. Chinese Journal of Infection and Chemotherapy, 19(4): 381-385.

[5] Zhang D, Zheng R, 2019, Preface: Pay Attention to the Progress of Diagnosis and Treatment of Senile Bloodstream Infection. Practical Geriatrics, 33(3): 209-210.

[6] Xie X, Sheng Y, Gao B, et al., 2012, Drug Resistance Analysis and Treatment Strategy of Nonfermenting Bacteria in ICU. Chinese Journal of Nosocomiology, 22(24): 5615-5617.

[7] Li Q, Cai T, Chang Y, 2010, Application of Microarray Technology in the Detection of Clinical Pathogenic Bacteria. Modern Practical Medicine, 22(5): 487-500. 